\title{
Surfaces Mimicking Glycosaminoglycans Trigger Different Response of Stem Cells via Distinct Fibronectin Adsorption and Reorganization
}

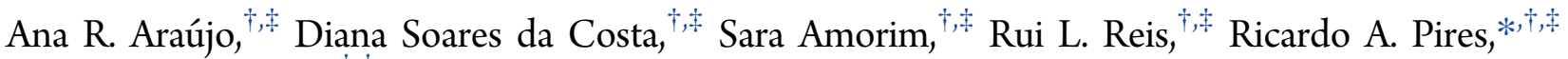 \\ and Iva Pashkuleva*, ${ }^{\dagger},+$ \\ †3Bs Research Group-Biomaterials, Biodegradables and Biomimetics, University of Minho, Headquarters of the European Institute \\ of Excellence on Tissue Engineering and Regenerative Medicine, AvePark, 4805-017 Taipas, Guimarães, Portugal \\ ${ }^{\sharp}$ ICVS/3B’s-PT Government Associate Laboratory, University of Minho, Braga/Guimarães, Portugal
}

\section{Supporting Information}

ABSTRACT: We report on the utility of a platform created by self-assembled monolayers to investigate the influence of the degree of sulfation of glycosaminoglycans (GAGs) on their interactions with fibronectin (Fn) and the impact of these interactions on the adhesion and morphology of human adipose derived stem cells (ASCs). We used the label-free QCM-D, AFM and SPR to follow the changes in the protein adlayer in close proximity to the substrates surface and QCM$\mathrm{D}$ in combination with live imaging to characterize the adherent cells. Our results suggest that Fn interactions with GAGs are governed by both H-bonding and electrostatic forces. Strong electrostatic interactions cause irreversible change in the protein conformation, while the weaker H-bonding only partially restricts the protein flexibility, allowing Fn reorganization and exposure of its binding sites for ASC adhesion. These findings imply that a delicate balance between these two types of forces must be considered in the design of biomaterials that mimic GAGs.

KEYWORDS: atomic force microscopy (AFM), multiparametric surface plasmon resonance (MP-SPR), self-assembling monolayers, adipose-derived stem cells, quartz crystal microbalance with dissipation (QCM-D)

\section{INTRODUCTION}

The extracellular matrix (ECM) comprises the closest cellular environment: it provides physical support for cells but also displays significant biochemical information via the molecules secreted by them. ${ }^{1-3}$ Although the chemical composition, organization, and turnover are unique for each cell, ECM of different tissues/cells share some common components, namely proteins and their glycoconjugates (proteoglycans and glycoproteins). Multivalent, supramolecular interactions between these insoluble macromolecules with soluble bioactive counterparts regulate the proper function of the ECM and thus, the development, function and homeostasis of all eukaryotic cells. ${ }^{2}$ Besides the importance of these interactions, their study is quite complex not only because they are weak and therefore difficult to measure, but also because of the complex environment in which they occur. Previously, we have developed model surfaces that mimic glycosaminoglycans (GAGs) and we have validated them in studies with cells and growth factors. ${ }^{4,5}$ These substrates are created by self-assembly of alkanethiols with $-\mathrm{SO}_{3} \mathrm{H}$ and $-\mathrm{OH}$ end groups-the main functionalities that are involved in GAGs supramolecular interactions with other bioentities in the physiological milieu. The main advantages of these surfaces are preparation simplicity, well-defined surface chemistry, and reproducibility. ${ }^{5,6}$ Moreover, the presentation of the functional groups on the surface (no steric hindrance) makes them available for multivalent interactions with an analyte and the compatibility with different characterization techniques (including label-free approaches, such as surface plasmon resonance, SPR, and quartz crystal microbalance, QCM-D) allows the measurement of these interactions. Indeed, single-component or mixed selfassembled monolayers (SAMs) have been widely used to elucidate the effect of surface chemistry, hydrophobicity and/or charge on protein adsorption and the following cellular behavior. $^{7-14}$

In this study, we used SAMs to mimic GAGs with different sulfation degree (simulated by mixing alkanethiol with $-\mathrm{SO}_{3} \mathrm{H}$ and $-\mathrm{OH}$ end groups at different ratios) and to characterize in situ their interactions with another ECM componentfibronectin (Fn). Because Fn plays a main role in cell adhesion and growth, we have further investigated the impact of the substrate-protein interactions on the cellular behavior using adipose derived stem cells (ASCs). The use of ASCs have a growing impact in tissue engineering and regenerative medicine fields because of several advantages that they have, such as, multipotential differentiation (similar to bone marrow stem

Received: April 14, 2016

Accepted: October 7, 2016 
cells), simpler isolation, and much easier access to subcutaneous adipose tissue when compared to bone marrow. ${ }^{15}$

\section{EXPERIMENTAL SECTION}

Chemicals. Unless otherwise stated, chemicals were purchased from Sigma-Aldrich and used without further purification. Human plasma fibronectin (Fn) was purchased from Millipore Iberica.

Materials. Gold-coated quartz crystals (QSX301, Q-Sense, Sweden) were used for the QCM-D measurements. Gold-coated sensors (BioNavis, Finland) were used for the SPR characterization. The AFM images were acquired using RFESP tips (Bruker, Germany) with a spring constant of $3 \mathrm{~N} / \mathrm{m}$ and a resonance frequency of $75 \mathrm{kHz}$. The substrates used for the live imaging were glass slides uniformly coated with gold $(20 \mathrm{~nm})$ by the e-beam physical vapor deposition (ATC Orion series UHV Evaporation system, AJA International Inc.). Titanium (3-5 nm film) was used as a primer improving the adhesion between the gold and the glass. All the substrates were cleaned (piranha solution, $10 \mathrm{~min}$ ) prior use. The single component and mixed SAMs (Figure S1) were formed on the substrates as previously described. ${ }^{5}$ Briefly, the cleaned substrates (QCM crystals, SPR sensors, and gold-coated glass) were immersed in $20 \mathrm{mM}$ ethanol solution of $\mathrm{HS}\left(\mathrm{CH}_{2}\right)_{11} \mathrm{OH}$ (samples designated as $\left.\mathrm{SO} 3 \mathrm{H} 0\right), \mathrm{HS}\left(\mathrm{CH}_{2}\right)_{11} \mathrm{SO}_{3} \mathrm{H}$ (synthesized in our lab, ${ }^{5}$ designated as $\mathrm{SO} 3 \mathrm{H} \mathrm{100)}$ or mixture of these at $-\mathrm{SO}_{3} \mathrm{H}:-\mathrm{OH}$ ratios of $1: 3$ and $3: 1$ in order to obtain 25 and $75 \%$ of $-\mathrm{SO}_{3} \mathrm{H}$ groups on the surface (samples referred to as $\mathrm{SO} 3 \mathrm{H} 25$ and $\mathrm{SO} 3 \mathrm{H} 75$, respectively). After $48 \mathrm{~h}$, the coated substrates were washed several times with ethanol, dried under $\mathrm{N}_{2}$, and immediately used in the following studies.

Adipose-Derived Stem Cells (ASCs). ASCs were isolated from human subcutaneous adipose tissue (age range between 20 and 36 years) obtained from lipoaspiration procedures under the scope of a cooperation agreement with Hospital da Prelada (Porto, Portugal). Aspirates were washed with PBS containing 10\% Antibiotic/ Antimycotic and then the adipose tissue was digested with a phosphate buffer saline (PBS) solution containing $0.1 \%$ collagenase from Clostridium histolyticum (Sigma-Aldrich) during $45 \mathrm{~min}$ at $37^{\circ} \mathrm{C}$, under stirring. The digested tissue was gently pressed through a strainer and centrifuged at $1000 \mathrm{~g}$ for $10 \mathrm{~min}$. The cell pellet was incubated in lysis buffer $\left(155 \mathrm{mM} \mathrm{NH}_{4} \mathrm{Cl}, 5.7 \mathrm{mM} \mathrm{K}_{2} \mathrm{HPO}_{4}, 0.1 \mathrm{mM}\right.$ EDTA) for $10 \mathrm{~min}$ to remove erythrocytes and then centrifuged at 800 $g$ for $10 \mathrm{~min}$. Cells were expanded in $\alpha$-modified Eagle's medium (Sigma-Aldrich) supplemented with $1 \%$ Antibiotic/Antimycotic (Gibco) and 10\% Fetal Bovine Serum (FBS, Gibco).

Real Time Monitoring of Fn Adsorption by Multiparametric Surface Plasmon Resonance (MP-SPR). Fn adsorption was followed in real time with a multiparametric instrument SPR Navi 200 (BioNavis, Finland), equipped with two lasers (670 and $785 \mathrm{~nm}$ ) in both measurement channels. All measurements were performed using a full angular scans. The protein adsorption was performed in situ by injecting Fn $(10 \mu \mathrm{g} / \mathrm{mL}$ in a serum free $\alpha$-MEM) at a rate of 50 $\mu \mathrm{L} / \mathrm{min}$. Upon signal stabilization, a washing step with a serum free $\alpha$ MEM was carried out and the liquid flow was replaced by air. The measurements in air were performed immediately after changing the media (detected by a pronounced shift in the resonance curves to lower angles) to guarantee the preservation of the protein organization. The spectra in $\alpha$-MEM and in air, both at 670 and $785 \mathrm{~nm}$, were fitted using the Winspall software (version 3.02, Max Planck Institute for Polymer Research, Mainz, Germany). SPR angular scans of bare sensor surfaces were simulated first, and the parameters obtained in the fitting of the first layers (glass-chromium-gold) were used in further simulations with the films. The simultaneous determination of both thickness and refractive index (RI) was performed following previously described procedure. ${ }^{16,17}$

Characterization of the Adsorbed Fn by Atomic Force Spectroscopy (AFM). The substrates used in the MP-SPR experiments were removed from the SPR chamber and further analyzed with an atomic force microscope NanoWizard 3 (JPK Instruments, Germany). The measurements were performed in air-dry state in AC mode. Different areas of each substrates with dimensions of $5 \times 5 \mu \mathrm{m}^{2}$ and $1 \times 1 \mu \mathrm{m}^{2}$ were scanned in order to have representative data. The data were analyzed with the JPK data processing software.

Real Time Characterization of Protein Adsorption by Quartz Crystal Microbalance with Dissipation (QCM-D). The QCM-D experiments were performed with E4 instrument (Q-Sense, Sweden). All the assays were performed at $37{ }^{\circ} \mathrm{C}$ and thus, the used solutions were equilibrated at this temperature before being introduced into the measurement chamber. The QCM-D crystals with the SAMs were placed in the QCM-D flow chamber and a stable baseline was acquired by flowing a serum free $\alpha$-MEM. The Fn $(10 \mu \mathrm{g} / \mathrm{mL}$ in a serum free $\alpha$ MEM) was then added at flow rate of $50 \mu \mathrm{L} / \mathrm{min}$. Upon stabilization of the signal, the flow was stopped for $30 \mathrm{~min}$ to allow the protein to adsorb. Finally, the sensors were rinsed with $\alpha$-MEM to remove loosely bound material. The resonance frequency shift, $\Delta f$, and the dissipative shift, $\Delta \mathrm{D}$, were recorded at several harmonics $(n=3,5,7$, 9, 11, and 13). The Voigt model was applied to calculate the adsorbed protein mass, using two overtones (seventh and ninth).

In Situ Characterization of Cell Adhesion by Quartz Crystal Microbalance with Dissipation (QCM-D). Immediately after the Fn deposition in the QCM-D (the step described above), ASCs $(0.11 \times$ $10^{6}$ cells $/ \mathrm{mL}$ ) were introduced into the chamber at a flow rate of 150 $\mu \mathrm{L} / \mathrm{min}$. Upon signal change, the flow was stopped for $1 \mathrm{~h}$ to allow cell attachment. Then, the sensors were rinsed with serum free $\alpha$-MEM at a rate of $300 \mu \mathrm{L} / \mathrm{min}$. The obtained data are presented as $\Delta D / \Delta f(3 \mathrm{rd}$ overtone) plots.

Characterization of Cell Spreading by Live Imaging. The substrates used in these experiments were gold coated glasses modified with single component and mixed SAMs. ASCs $\left(6000 \mathrm{cells} / \mathrm{cm}^{2}\right)$ were seeded (serum free $\alpha$-MEM) on these substrates under standard conditions either in the absence of Fn or on substrates previously coated with Fn by incubation with protein solution in serum free $\alpha$ MEM $(10 \mu \mathrm{g} / \mathrm{mL})$ at $37{ }^{\circ} \mathrm{C}$ for $30 \mathrm{~min}$. Live monitoring of cell spreading was performed at $37{ }^{\circ} \mathrm{C}$ in an inverted microscope (Zeiss Axio Observer) equipped with a temperature and $\mathrm{CO}_{2}$ control device $\left(5 \% \mathrm{CO}_{2}\right)$. Time-lapse images $(20 \mathrm{X})$ were captured every $1 \mathrm{~min}$ using Zen software. Cells were continuously observed for $5 \mathrm{~h}$. Image stacks were analyzed with image processing software Fiji (http://fiji.sc/wiki/ index.php/Fiji). The typical cell behavior for each substrate is shown by images taken at $5 \mathrm{~min}$ intervals and up to $45 \mathrm{~min}$ culture time as the most significant changes in the cell spreading were observed immediately after seeding.

Cell Characterization by Immunocytochemistry. Cytoskeleton organization and formation of focal adhesion was visualized for cells studied by QCM-D $(1 \mathrm{~h})$ and live imaging $(1$ and $3 \mathrm{~h}$ ). The substrates with the cultured cells were removed from the respective equipment, washed twice with PBS, fixed with $10 \%$ neutral buffered formalin for 30 min at $4{ }^{\circ} \mathrm{C}$, permeabilized with $0.1 \%$ Triton X-100 in PBS for 5 min, and blocked with $3 \% \mathrm{BSA}$ in PBS for $30 \mathrm{~min}$ at room temperature. Cytoskeleton organization was visualized by phalloidinTRITC conjugate (1:200 in PBS for $30 \mathrm{~min}$, Sigma). Nuclei were counterstained with 4,6-diamidino-2-phenylindole (DAPI; 1:200 in PBS for $30 \mathrm{~min}$ ). Formation of actin cap and normal focal adhesions was assessed using a primary antibody against paxillin (1:250 in 1\% w/ v BSA/PBS, VWR), followed by donkey antirabbit Alexafluor-488 (1:500 in $1 \% \mathrm{w} / \mathrm{v}$ BSA/PBS, Alfagene). Samples were washed with PBS, mounted with Vectashield (Vector) on glass slides and observed under an Imager Z1 fluorescence microscope (Zeiss). Photographs were taken with an Axio Cam MRm (Zeiss).

Statistical Analysis. The normality of the data was evaluated using Shapiro-Wilk test $(p<0.05)$. When the data did not follow a normal distribution an initial Kruskal-Wallis test was executed followed by Mann-Whitney test. In all cases, significant variations are marked with (*) for $p<0.001$.

\section{RESULTS AND DISCUSSION}

Fibronectin (Fn, Figure S2) is a large size (440 kDa), ubiquitous ECM glycoprotein that can be found around all types of cells and throughout all stages of life. It has several 

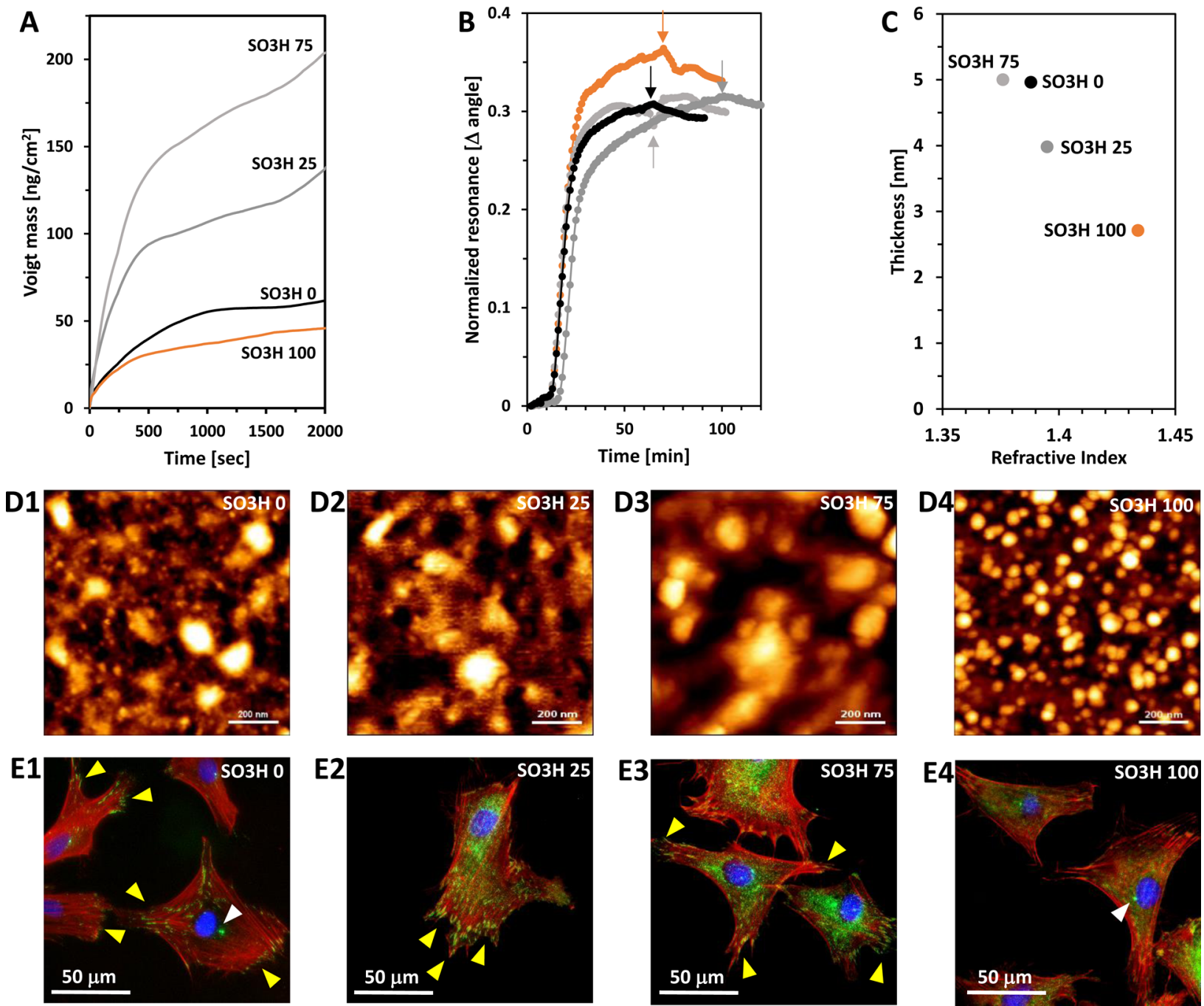

Figure 1. Adsorption profiles of Fn on different surfaces characterized by (A) QCM-D, (B, C) SPR, and (D) AFM and (E) the respective cellular response. The sensograms (B) were recorded in $\alpha$-MEM at wavelength of $670 \mathrm{~nm}$; the arrows indicate the washing step. At the end of the adsorption process, the protein layer was characterized by two-color, two-media (C) MP-SPR and (D) AFM. The immunostaining of the adipose-derived stem cells (ASCs) was performed after recording their behavior by QCM-D ( $1 \mathrm{~h}$ on Fn-coated substrates). Immunostaining of paxillin (green), actin (red), and nuclei (blue). White arrows indicate the centrosomes and yellow arrows show the focal adhesions. Images of the control samples (ASCs on QCM-D crystals without Fn) are provided in Figure S7.

domains (Figure S2A) allowing simultaneous binding to cells and other ECM components, namely GAGs, other Fn molecules, and other ECM proteins. ${ }^{1,18,19}$ Whereas in solution it has a compact globular conformation with a diameter of 16$35 \mathrm{~nm},{ }^{20}$ around the cells, Fn is organized in a network of fibers with a diameter of $2 \mathrm{~nm}$ and a length that can very between 120 and $180 \mathrm{~nm} .^{3,18,20}$ The assembly of this network is essential for cells and depends on the Fn dimerization via intramolecular disulfide bonds (Figure S2B) and the multiple interactions of these dimers with integrins (e.g., $\alpha 5 \beta 1$ binds to the RGD domain) and with other Fn molecules. It must be noticed that some of the Fn binding sites also have heparin (Hep) binding activity which indicates the involvement of this GAG in cooperative events influencing the interactions of Fn with other ligands. As an example, Hep binds reversibly to Fn type III (Figure S2) inducing conformational change that is preserved even after the Hep unbinding. ${ }^{21}$ The induced extended conformation reveals new binding sites for growth factors (e.g., VEGF) and thus, increases dramatically the affinity of Fn for these biomolecules. ${ }^{21,22}$ Noteworthy, this interaction is specific for Hep as other sulfated GAGs such as chondroitin sulfate or desulfated Hep do not alter the Fn conformation. ${ }^{21}$

Previous studies have demonstrated that Fn affinity for Hep is relatively low $\left(\mathrm{K}_{\mathrm{D}}\right.$ about $0.1-1 \mu \mathrm{M}$, about $10-1000$ folds weaker than the interaction with FGF-2) and based mainly on electrostatic interactions. ${ }^{23-25}$ We have therefore created surfaces with an increasing charge density by mixing at different ratios alkanethiols with end groups representing the functionalities present in the GAGs molecules: $-\mathrm{OH}$ groups (polar groups, able to participate in $\mathrm{H}$-bonding but with no charge) and $-\mathrm{SO}_{3} \mathrm{H}$ groups (negative charge, able to participate in both $\mathrm{H}$-bonding and electrostatic interactions). The selfassembly of these alkanethiols (Figure S1, single component or mixtures) result in the formation of homogeneous SAMs with different charge (Table S1). ${ }^{4,5}$

Next, we investigated in situ the interaction of Fn with these surfaces using QCM-D and MP-SPR. QCM-D is an acoustic method in which the material deposition and/or increasing of a film thickness affects the propagation of a wave through a sensor (quartz crystal disk) and causes changes of its resonance 

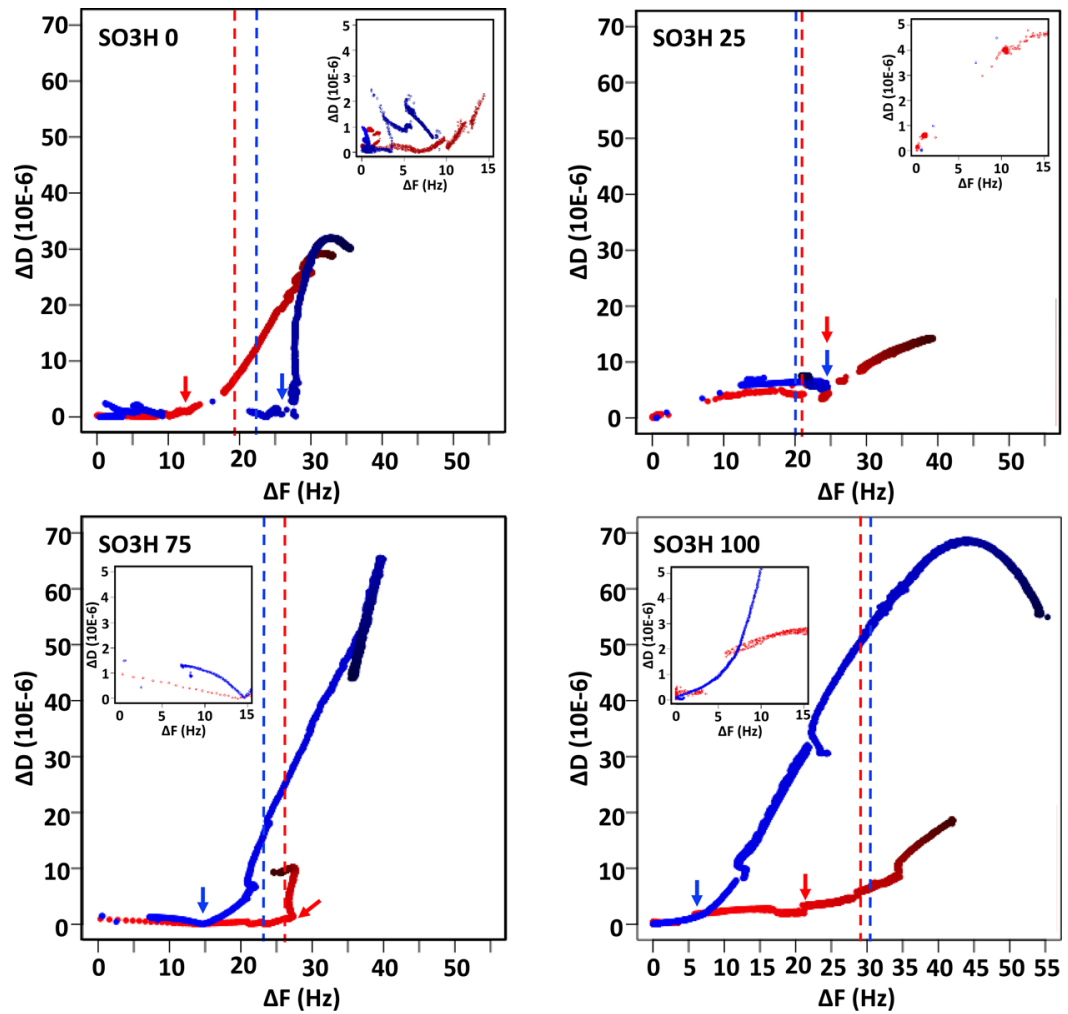

Figure 2. Representative $\Delta D / \Delta f$ plots (3rd overtone) showing changes during adhesion of ASCs onto SAMs' surfaces with different concentration of $-\mathrm{SO}_{3} \mathrm{H}$ groups in the presence (blue points) and in the absence (red points) of Fn on the sensor surface. The broken lines show the points at which the cell flow was stopped. The time is indicated by a color code: pale color indicates the beginning of the process, whereas darker nuances are associated with its end. Arrows indicate the end of the cell seeding process. The insets represent amplified data plots for the beginning of the process.

frequency $(\Delta f)$. Acoustic contrast is largely affected by coupled water and/or conformational changes. Change in the energy dissipation $(\Delta D)$ reflects this sensitivity and is measured by continuous switch on and off of the driving potential resulting in the damping of the oscillatory motion. On the other hand, SPR is an optical detection method whose contrast is determined by changes in the RI caused by the deposited film. The SPR signals are therefore proportional to the adsorbed mass and affected minimally by the hydration of the adsorbed entity. We complement these real time characterization techniques with AFM analyses. The space resolution of AFM is much higher in comparison with other microscopies (e.g., electron microscopy) as the force microscopy does not use lenses and thus, the diffraction and aberration are not limiting its spatial resolution. We therefore expected that the combination of these three label-free techniques will give us detailed information about the protein adsorption in terms of quantity, homogeneity, and conformation.

Our results demonstrated that Fn adsorbs onto all surfaces (Figure 1). Both real time techniques (QCM-D and SPR) showed a rapid initial adsorption that is slowing as the surface coverage increases (Figures 1A, B). The QCM-D and SPR signals do not change upon washing of the adsorbed protein with a buffer indicating stable interactions of $F n$ with the underlying surface. The quantity of the adsorbed protein is different for the studied surfaces. The QCM-D data showed an increase of the adsorbed mass with the augmentation of the $-\mathrm{SO}_{3} \mathrm{H}$ concentration - a maximum was reached for the $\mathrm{SO} 3 \mathrm{H}$ 75 surface and then a drop to a minimum for the surface formed by self-assembly of $\mathrm{HS}\left(\mathrm{CH}_{2}\right)_{11} \mathrm{SO}_{3} \mathrm{H}$ alone was measured (Figure 1A). The results obtained for the single- component systems (SO3H 0 and $\mathrm{SO} 3 \mathrm{H} \mathrm{100)}$ are in good agreement with previous reports on the quantification of Fn adsorption on surfaces with defined chemistry using alternative characterization methodologies, namely protein radiolabeling, ${ }^{8}$ conjugation to fluorophores ${ }^{26,27}$ and Western blot characterization. ${ }^{9,10}$ All these studies refer to $-\mathrm{OH}$ functionalized surfaces as substrates with lower quantity of adsorbed $\mathrm{Fn}$ as compared with the hydrophobic $-\mathrm{CH}_{3}{ }^{8-10,27}$ and the positively charged $-\mathrm{NH}_{2}{ }^{8,12}$ Moreover, our data are also in excellent agreement with study by Groth et al., who measured similar quantity of $\mathrm{Fn}$ adsorbed on $-\mathrm{OH}$ and $-\mathrm{SO}_{3} \mathrm{H}$ functionalized surfaces with slight prevalence of protein on the $-\mathrm{OH}$ surface. $^{12}$

The results obtained from them are striking alike to the reported herein, although the authors have used different methodology for quantification of the adsorbed protein, namely ELISA assays, and screened single component SAMs formed on silanized glass surfaces. Noteworthy, different behavior of $-\mathrm{SO}_{3} \mathrm{H}$ groups as compared with other negatively charged groups (e.g., $-\mathrm{COOH}$ ) is reported in the same study: greater adsorption capacity for $\mathrm{Fn}$ and increased cell adhesion is demonstrated for the $-\mathrm{SO}_{3} \mathrm{H}$ functionalities. In fact, we have also observed very similar tendency as the described above (Figure 1A) when studying the FGF-2 binding affinity of these substrates (both mixed and single component SAMs). ${ }^{4}$ We have demonstrated that the bias behavior of the $\mathrm{SO} 3 \mathrm{H} 100$ is not due to the very high content of $-\mathrm{SO}_{3} \mathrm{H}$ groups but rather to the lack of $-\mathrm{OH}$ groups on these surfaces and their contribution to the adsorption via H-bonding.

As the deposited mass can also include water, we further analyze the Fn adsorption with MP-SPR and AFM. We have 
used the two-color SPR approach (wavelengths of 670 and 785 $\mathrm{nm})$ combined with two media ( $\alpha$-MEM and air) measurements to determine simultaneously the RI and the thickness of the deposited layer, i.e., we did not use any estimated or assumed values for the calculations (Figure 1C). ${ }^{16,17}$ The water content and the protein conformation can differ for the Fn adsorbed on the studied substrates and these differences can affect the RI. Therefore, the use of estimated RI values commonly applied for thickness calculations can lead to inaccurate results as they do not reflect this change. Our results demonstrated that while all RI values are near to the used estimated RI for Fn $\left(1.4^{20}\right)$, substrate chemistry indeed affect the RI of the adsorbed layer (Figure $1 \mathrm{C}$ ). It is noteworthy mentioning that the only surface, which does not contain $-\mathrm{OH}$ groups, affect mostly the RI of the adsorbed Fn. This surface chemistry was also associated with the deposition of the thinnest Fn layer $(2.7 \mathrm{~nm})$. Previous study has demonstrated that nonspecific Fn adsorption on $\mathrm{TiOx}$ surface results in formation of a layer with thickness of about $6 \mathrm{~nm}$ while the specific adsorption (biotinylated Fn adsorbed on streptavidin monolayer) corresponds to a layer with thickness of $2 \mathrm{~nm}$ in which the Fn is in its linear conformation. ${ }^{20}$ The obtained by us values are within these limits although they are different for each studied substrate (Figure 2C). We therefore have speculated that conformational changes induced upon the protein adsorption can occur. Indeed, the QCM-D derivative plot of the adsorption profiles (Figure S3) demonstrated that the process is complex for the surfaces containing both functional groups ( $\mathrm{SO} 3 \mathrm{H} 25$ and $\mathrm{SO} 3 \mathrm{H} \mathrm{75)}$ ). This stepwise process can be explained with an initial rapid absorption, followed by reorganization of the adsorbed protein on the surface that allows further protein molecules to adsorb on the resulted free area. ${ }^{28}$ In fact, the adsorption profile of $\mathrm{Fn}$ for these two substrates does not reach a plateau (Figure $1 \mathrm{~A}$ and Figure S3) demonstrating that the process of reorganization and additional adsorption is still ongoing. The $\mathrm{SO} 3 \mathrm{H} 0$ and SO3H 100 surfaces behave different-a simple adsorption profile was observed for these substrates (Figure S3). The SPR data, however, demonstrated that the thickness of the Fn layer on these substrates is quite different. In fact, there is a great deal of research evidencing that substrates surface properties can influence not only the quantity of the adsorbed proteins but also their conformation. $8,9,27,29,30$ The most studied surface property influencing Fn conformation is the wettability: hydrohpobic surfaces induce material-driven Fn fibrillogenesis, whereas globular conformation is typical for the protein adsorbed on hydrophilic surfaces. ${ }^{9,10,27,30,31}$ Our results are in good agreement with these previous reports. All surfaces studied by us are hydrophilic (Table S1) and the AFM analysis indeed revealed the expected formation of aggregates of different sizes on all $-\mathrm{OH}$ containing surfaces (Figure 1D13 ). We did not detect any material-induced Fn fibrillogenesis. However, very different organization of the Fn was observed on the SO3H 100 substrates (Figure 1D4): the formed layer was homogeneous and composed by individual Fn molecules but not aggregates (size of about $1 \mathrm{~nm} \times 30 \mathrm{~nm}$ has been previously reported for globular $\mathrm{Fn}^{20}$ ). This peculiar $\mathrm{Fn}$ organization is most probably a result of strong electrostatic interactions between the substrate and the protein, which overpower the supramolecular forces that drive the protein selfassociation. This result is in line with previously reported single-molecule AFM measurements showing globular but extended Fn conformation upon its adsorption in the presence of Hep (the biomolecule with the highest negative charge that exist in Nature). ${ }^{21}$ This conformational change is related to the sulfate groups as the presence of either chondroitin sulfate (less sulfated than Hep) or the desulfated Hep derivative does not affect the Fn conformation.

Conformational changes can encrypt hidden binding domains $^{21,32}$ and thus in our next experiments we used ASCs to evaluate the effect of the substrate-protein interactions on the cell attachment process. It is well-documented that upon adhesion cells reorganize the Fn into fibrillar matrices by a complex multistep process involving interactions of the protein with activated integrins on the cell surface. ${ }^{10,18,31,33,34}$ This process often involve inceptive removal of the preadsorbed $\mathrm{Fn}^{26,31,35}$ and thus, it depends on the chemistry of the substrate and the respective adsorption force. ${ }^{31}$ Altered protein conformation triggered by different surface chemistry also affect the integrin-Fn interactions and the following reorganization of the protein. ${ }^{10,36}$ Indeed, Garcia et al. have demonstrated that Fn-coated SAMs are integrin specific: Fn on negatively charged surface $(-\mathrm{COOH})$ supports both $\alpha_{\nu} \beta_{3}$ and $\alpha_{5} \beta_{1}$ binding while the $\mathrm{Fn}$ on $-\mathrm{OH}$ and $-\mathrm{NH}_{2}$ surfaces selectively recruits $\alpha_{5} \beta_{1}$ integrins. ${ }^{8}$

Herein, we used a combination of QCM-D, live imaging, and immunocytochemistry to assess the cellular interactions with the SAMs and the effect of preadsorbed $\mathrm{Fn}$ on these interactions. The reason for using QCM-D with microscopy is that cells that look very similar under microscope can have quite different interfacial and mechanical properties and thus, their characterization requires combination of a microscopy with another method. On the other hand, QCM-D is sensitive only to changes that occur very near $(\mathrm{nm})$ to the sensor surface because of the penetration depth of the QCM-D shear wave (the typical penetration depth of the shear acoustic waves in water is about $0.25 \mu \mathrm{m}$ at $f=5 \mathrm{MHz}^{37}$ ). Keeping in mind this peculiarity of the QCM-D technique, five processes that can influence the signal have been suggested: (i) the initial physical contact (cell seeding on the sensor surface) leading to the first QCM response; (ii) secretion of ECM components; (iii) cell spreading; (iv) modification of the adhesion properties at the interface, e.g., strength of adhesion; and (v) changes in the cytoskeleton. ${ }^{38}$ Although the first two processes significantly affect the $\Delta f$, the last ones are reflected mainly in the magnitude of $\Delta D$. It must be noticed that some of these processes often occur simultaneously, which makes the interpretation of the QCM-D data quite complex.

We present the QCM-D results as $\Delta D / \Delta f$ plots (Figure 2). This kind of presentation visualize readily the structural properties of the biolayers deposited on the sensor (e.g., adherent cells) and it is qualitatively independent of the number and spatial distribution of the deposits on the sensor as the shape of the lateral sensitivity variation for $\Delta D$ and $\Delta f$ are the same. The slopes in these plots represent the dynamics of the adhesion process and its different stages and thus, they are unique (but reproducible) for each type of cell and substrate and can be used to compare different systems. ${ }^{39}$ Because of their specificity, these plots are often called cellular acoustic fingerprints. ${ }^{37-39}$

The comparison between the plots for different surfaces in the absence of preadsorbed Fn (Figure 2, red lines) demonstrates the presence of at least two slopes with quite different shape, indicating different adhesion process/cellular behavior. The first slope is characterized by a fast (insets on Figure 2: low density of points in $\Delta D / \Delta f$ plots reveals fast 

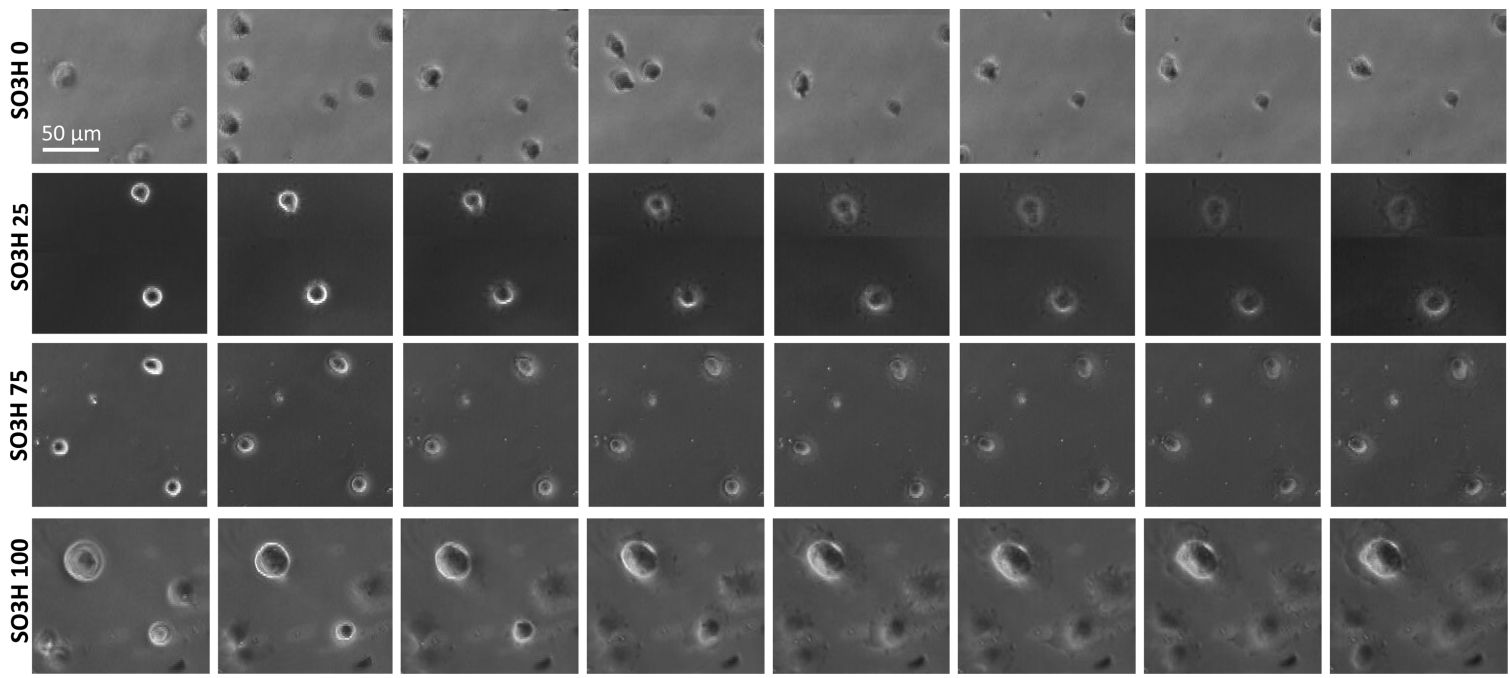

Figure 3. Live imaging of adipose-derived stem cells adhering and spreading on the studied substrates without Fn adsorption. The first image was taken $5 \mathrm{~min}$ after the cell seeding and the following ones at intervals of $5 \mathrm{~min}$ up to $40 \mathrm{~min}$.
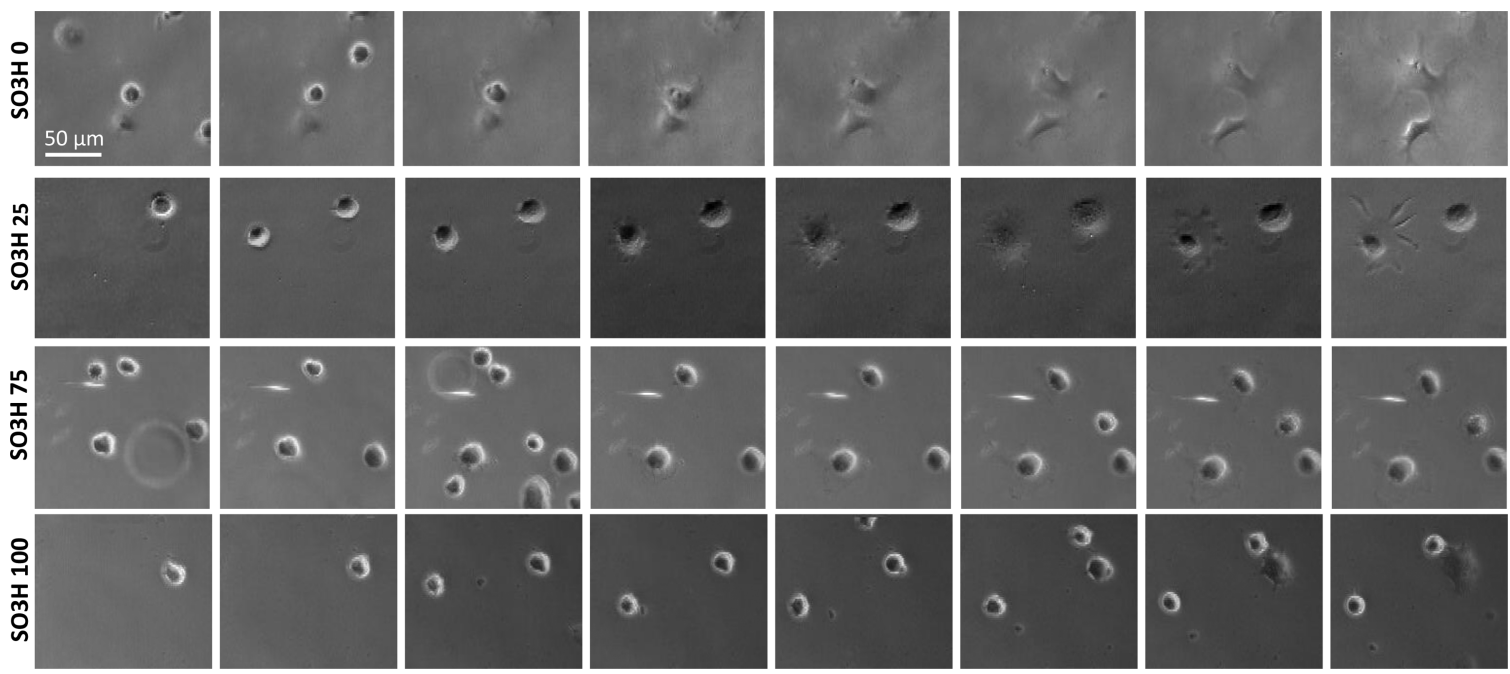

Figure 4. Live imaging of adipose-derived stem cells seeded on the studied substrates after Fn adsorption. The first image was taken 5 min after the cell seeding and the following ones at intervals of $5 \mathrm{~min}$ up to $40 \mathrm{~min}$.

kinetics) and significant increase of $\Delta \mathrm{f}$ with almost no change of $\Delta D$ and it is associated with the initial cell seeding on the sensors (the end of this process is identified with arrows in Figure 2). After this process, an increasing in the dissipation accompanied by mass deposition was observed for all surfaces. The absolute values of these changes in $\Delta D$ and $\Delta f$ are different for all the studied surfaces (Figure S6). As mentioned above, the mass deposition at this stage is associated with secretion of proteins and other ECM components, while changes in the dissipation are related with either remodeling of the deposited ECM components or with the mechanical properties of the seeded cells caused by their interactions with the substrates/ sensors. The largest $\Delta D$ was measured for the $\mathrm{SO} 3 \mathrm{H} 0$ surface and we associated it with relative weak cellular adhesion and spreading. When the surfaces have $-\mathrm{SO}_{3} \mathrm{H}$ groups, the absolute value of the $\Delta D$ is $2-3$ fold smaller, indicating that these groups induce different shear elasticity of the cytoskeleton most probably as a result of stronger adhesion. These results are in excellent agreement with the ASCs live imaging, which confirmed that in the absence of preadsorbed $\mathrm{Fn}$, the presence of the $-\mathrm{SO}_{3} \mathrm{H}$ groups on the surface induces fast adhesion and spreading of ASCs and this is particularly evident in the case of SO3H 100 substrates (Figure 3). The immunocytochemistry of the ASCs (Figure S7) is also in line with QCM-D and live imaging outputs: we can observe spread cells on $\mathrm{SO} 3 \mathrm{H} 100$ substrates and round ones on the $-\mathrm{OH}$ functionalized substrates.

The preadsorbed Fn induces a tremendous difference in QCM-D signals (Figure 2, blue lines and Figure S6) especially for the $-\mathrm{SO}_{3} \mathrm{H}$ rich surfaces ( $\mathrm{SO} 3 \mathrm{H} 75$ and $\mathrm{SO} 3 \mathrm{H} 100$ ). Similar large dissipation shifts have been previously observed for different surfaces and cells in the presence of serum or individual serum proteins. ${ }^{39-41}$ However, there is no consensus in the literature about the magnitude of this change and the reasons behind it. ${ }^{37}$ As mentioned above, cells attached to the sensor surface form acoustically thick layer (up to $15 \mu \mathrm{m}$ in the absence of preadsorbed Fn, Figure 4B2). In the case of preadsorbed protein this layer is even thicker (for QCM-D) with the cells placed above the Fn. We therefore suggest that the main reason behind the observed changes is the abovementioned reorganization of the preadsorbed Fn (that is nearest to the sensor surface and form a layer whose thickness 
is comparable with the penetration depth of the shear acoustic waves) by the attaching cells.

Previous studies have also suggested that the ECM layer between the cells and the sensor can play significant role in the QCM-D output signals. ${ }^{37,42}$ Particularly interesting and indicative are the results for the $\mathrm{SO} 3 \mathrm{H} 0$ substrate. In this case, we observed a large dissipation shift (Figure 2), with no accompanying frequency shift. This signal indicates no production of ECM but only reorganization of preadsorbed Fn. Only after reorganizing this layer, cells start to secrete ECM components (changes in $\Delta f$ are visible) and eventually spread (small change of $\Delta D$ ). These results are in very good agreement with a recent work of Luo et al., who have demonstrated that adsorption force of Fn on $-\mathrm{OH}$ SAMs is much weaker than on hydrophobic $\left(-\mathrm{CH}_{3}\right)$ and positively charged $\left(-\mathrm{NH}_{2}\right)$ surfaces. ${ }^{31}$ As a result, the protein is more flexible on $-\mathrm{OH}$ functionalized surfaces ( $\mathrm{SO} 3 \mathrm{H} 0$ ) and its cell binding domains (Figure 1) more accessible for interactions with integrins. ${ }^{8,31}$

The results are different for $\mathrm{SO} 3 \mathrm{H} 25$ substrate: after the initial signal for cell seeding, no further changes are detected either for the deposited mass nor for the energy dissipation. Indeed, for this surface chemistry, we have observed already reorganization of the Fn during the deposition (Figure S3) and although some further reorganization can occur, the impact on the $\Delta D$ is very small. In the case of the surfaces with higher content of $-\mathrm{SO}_{3} \mathrm{H}$ groups, we observe very large dissipation change concomitant with mass deposition. These signals demonstrate intensive ECM secretion and remodelling. We hypothesize that either cell binding domains of Fn are hindered for these substrates (e.g., by specific interactions between the closely positioned heparin binding domain of Fn (Figure S2) and $-\mathrm{SO}_{3} \mathrm{H}$ groups) or the multivalent electrostatic interactions between the preadsorbed $\mathrm{Fn}$ and the $-\mathrm{SO}_{3} \mathrm{H}$ rich substrates are strong enough and do not allow further reorganization of the protein by the cells. We therefore performed live imaging (Figure 4) to complement the QCM-D data but also to obtain additional information beyond the cell surface properties. This further characterization reveals an opposite effect of the preadsorbed Fn on single-component systems ( $\mathrm{SO} 3 \mathrm{H} 0$ and $\mathrm{SO} 3 \mathrm{H} \mathrm{100);} \mathrm{while} \mathrm{it} \mathrm{promotes} \mathrm{cellular}$ adhesion and spreading on $-\mathrm{OH}$ functionalized surfaces, it inhibits these processes on $-\mathrm{SO}_{3} \mathrm{H}$ functionalized ones (Figure 4 first vs last row).

In fact, this behavior is in agreement with the QCM-D data (Figure 2, beside the different absolute values the trend indicated by the red line of the $\mathrm{SO} 3 \mathrm{H} 0$ is similar to the blue line of the $\mathrm{SO} 3 \mathrm{H} 100$ and vice versa). In the absence of $-\mathrm{SO}_{3} \mathrm{H}$ groups ( $\mathrm{SO} 3 \mathrm{H} 0$ ), the cells recognize the preadsorbed $\mathrm{Fn}$, form initial attachment points via their integrins, reorganize the protein layer that is "hold" on the surface only by weak $\mathrm{H}$ bonding, and spread on the surface remodeled by them. On the $\mathrm{SO} 3 \mathrm{H} 100$ surface, cells either cannot recognize the Fn or they recognize it but, because of the strong electrostatic interactions, cannot reorganize it in order to induce functional integrin binding. When mixed SAMs are used $(\mathrm{SO} 3 \mathrm{H} 25$ and $\mathrm{SO} 3 \mathrm{H}$ 75) there is a balance between both trends.

These results were corroborated by immunocytochemistry (Figures $1 \mathrm{E}$ and 5 and Figure S7) and morphometric analysis (Figures S8 and S9) of ASCs cultured during the QCM-D analysis (Figure 1E and Figures S7 and S8) or at standard static culture conditions used for live imaging (no flow during the Fn adsorption and cell seeding) using gold coated slides as substrates and longer culture times (Figure 5 and Figure S9). The immunocytochemistry revealed another difference among
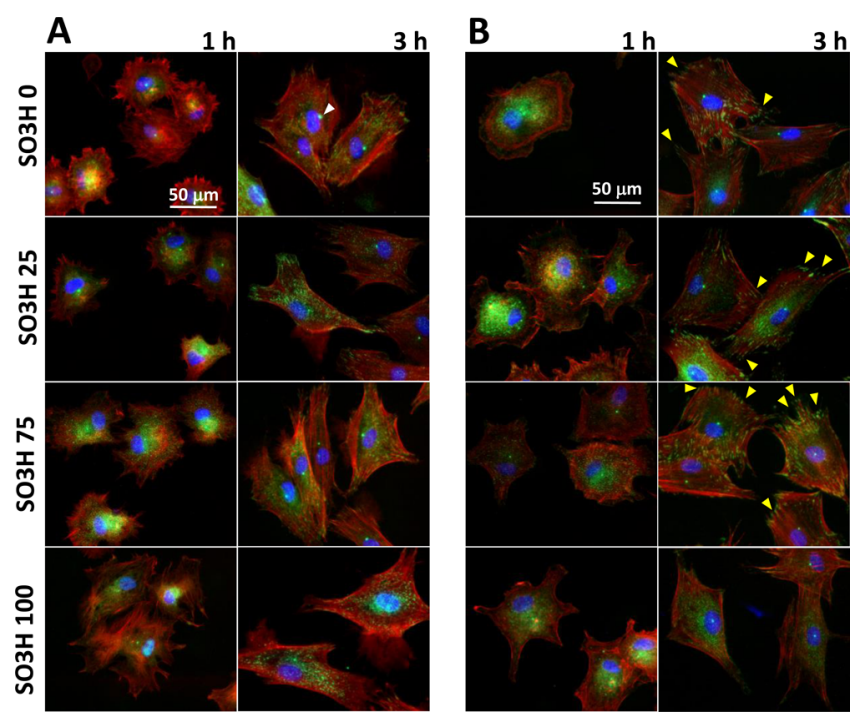

Figure 5. Representative fluorescence microscopy images of adiposederived stem cells cultured on single and mixed SAMs at two different time points: 1 and $3 \mathrm{~h}(\mathrm{~A})$ without and (B) with Fn. Immunostaining of paxillin (green), actin (red), and nuclei (blue). White arrow indicates centrosome and yellow arrows show the focal adhesions.

the studied substrates: in the presence of Fn, ASCs cultured on $-\mathrm{OH}$-containing surfaces $(\mathrm{SO} 3 \mathrm{H} 0, \mathrm{SO} 3 \mathrm{H} 25$, and $\mathrm{SO} 3 \mathrm{H} 75$; Figure 1E1-3 vs Figure S7 and Figure 5A vs Figure 5B) displayed organized actin stress fibers with prominent focal adhesion sites (FA). FAs are visible after $1 \mathrm{~h}$ when the ASCs were cultured in the QCM-D instrument (Figure 1E1-3, yellow arrows) and after $3 \mathrm{~h}$ at standard culture conditions (Figure 5B, yellow arrows) suggesting that the flow accelerates and/or amplifies cell response to the surface chemistry. We did not detect FAs at these time points for the ASCs cultured on $\mathrm{SO} 3 \mathrm{H} 100$ substrates in the presence of Fn. Altogether, these data suggest different adhesion mechanism of ASCs in response to surface-induced organization/conformational changes of Fn.

\section{CONCLUSIONS}

Our results demonstrate that QCM-D analysis provides unique information about the nanoscale interactions of cells with underlying surfaces. However, we suggest that in the presence of proteins and especially in the case of protein(s) preadsorbed on the substrate, the QCM-D data circumscribe mainly the reorganization of this protein layer, i.e., contribution of the cells' viscoelastic properties to the QCM signals is diminished because of the increased distance between the cells and the sensor.

We further demonstrate that tailored $-\mathrm{SO}_{3} \mathrm{H}$ concentration can be apply to finely tune environments around the cells by changing GAG-protein interactions. Our data suggest that fully sulfonated surfaces alter the bioactivity of proteins as Fn, by binding them stronger. The delicate balance between different supramolecular interactions (e.g., the H-bonding and electrostatic interactions discussed above) that govern the bioactivity and the responsiveness of the closest cellular environment should be considered in the design of biomaterials that mimic this milieu. 


\section{ASSOCIATED CONTENT}

\section{S Supporting Information}

The Supporting Information is available free of charge on the ACS Publications website at DOI: 10.1021/acsami.6b04472.

Data about formation and characterization of the used substrates; raw QCM-D and SPR data; AFM images at low magnification; and morphometric analysis of ASCs (PDF)

\section{AUTHOR INFORMATION}

\section{Corresponding Authors}

*E-mail: pashkuleva@dep.uminho.pt. Phone: +351 253510 908/7. Fax: +351253510909.

*E-mail: rpires@dep.uminho.pt.

\section{Author Contributions}

A.R.A. performed the cell culture and characterization experiments; D.S.C. isolated the ASCs and carried out the live imaging and morphometric analysis; S.A. performed the QCM$\mathrm{D}$ and SPR experiments; R.A.P. did the AFM analysis; R.L.R. provided facilities to perform the experiments. I.P. and R.A.P. conceived the project, interpreted the data and wrote the manuscript. All authors discussed the results, commented on the manuscript and have given approval to its final version.

\section{Notes}

The authors declare no competing financial interest.

\section{ACKNOWLEDGMENTS}

We acknowledge EU for financial support (FP7/2007-2013, under Grants REGPOT-CT2012-316331-POLARIS and ERC2012-ADG-20120216-321266 for the project ComplexiTE). I.P. is thankful to the Portuguese Foundation for Science and Technology (IF/00032/2013). The authors are very grateful to Dr. Raquel Teixeira for her help with the treatment and discussion of the MP-SPR data.

\section{ABBREVIATIONS}

GAGs, glycosaminoglycans

Fn, fibronectin

ASCs, adipose-derived stem cells

ECM, extracellular matrix

$\mathrm{RI}$, refractive index

SAMs, self-assembled monolayers

Hep, heparin

QCM-D, quartz crytal microbalance with dissipation monitoring

FA, focal adhesion

\section{REFERENCES}

(1) Mouw, J. K.; Ou, G. Q.; Weaver, V. M. Extracellular matrix assembly: a multiscale deconstruction. Nat. Rev. Mol. Cell Biol. 2014, 15 (12), 771-785.

(2) Azevedo, H. S.; Pashkuleva, I. Biomimetic supramolecular designs for the controlled release of growth factors in bone regeneration. Adv. Drug Delivery Rev. 2015, 94, 63-76.

(3) Singh, P.; Carraher, C.; Schwarzbauer, J. E. Assembly of Fibronectin Extracellular Matrix. Annu. Rev. Cell Dev. Biol. 2010, 26, 397-419.

(4) Amorim, S.; Pires, R. A.; Soares da Costa, D.; Reis, R. L.; Pashkuleva, I. Interactions between Exogenous FGF-2 and Sulfonic Groups: in Situ Characterization and Impact on the Morphology of Human Adipose-Derived Stem Cells. Langmuir 2013, 29 (25), 79837992.
(5) da Costa, D. S.; Pires, R. A.; Frias, A. M.; Reis, R. L.; Pashkuleva, I. Sulfonic groups induce formation of filopodia in mesenchymal stem cells. J. Mater. Chem. 2012, 22 (15), 7172-7178.

(6) Love, J. C.; Estroff, L. A.; Kriebel, J. K.; Nuzzo, R. G.; Whitesides, G. M. Self-assembled monolayers of thiolates on metals as a form of nanotechnology. Chem. Rev. 2005, 105 (4), 1103-1169.

(7) Lan, M. A.; Gersbach, C. A.; Michael, K. E.; Keselowsky, B. G.; Garcia, A. J. Myoblast proliferation and differentiation on fibronectincoated self assembled monolayers presenting different surface chemistries. Biomaterials 2005, 26 (22), 4523-4531.

(8) Keselowsky, B. G.; Collard, D. M.; Garcia, A. J. Surface chemistry modulates fibronectin conformation and directs integrin binding and specificity to control cell adhesion. J. Biomed. Mater. Res., Part A 2003, 66 A (2), 247-259.

(9) Llopis-Hernandez, V.; Rico, P.; Ballester-Beltran, J.; Moratal, D.; Salmeron-Sanchez, M. Role of Surface Chemistry in Protein Remodeling at the Cell-Material Interface. PLoS One 2011, 6 (5), e19610.

(10) Rico, P.; Hernandez, J. C. R.; Moratal, D.; Altankov, G.; Pradas, M. M.; Salmeron-Sanchez, M. Substrate-Induced Assembly of Fibronectin into Networks: Influence of Surface Chemistry and Effect on Osteoblast Adhesion. Tissue Eng., Part A 2009, 15 (11), 32713281.

(11) Keselowsky, B. G.; Collard, D. M.; Garcia, A. J. Integrin binding specificity regulates biomaterial surface chemistry effects on cell differentiation. Proc. Natl. Acad. Sci. U. S. A. 2005, 102 (17), 59535957.

(12) Altankov, G.; Richau, K.; Groth, T. The role of surface zeta potential and substratum chemistry for regulation of dermal fibroblasts interaction. Materialwiss. Werkstofftech. 2003, 34 (12), 1120-1128.

(13) Mrksich, M.; Whitesides, G. M. Using self-assembled monolayers to understand the interactions of man-made surfaces with proteins and cells. Annu. Rev. Biophys. Biomol. Struct. 1996, 25, $55-78$.

(14) Faucheux, N.; Schweiss, R.; Lutzow, K.; Werner, C.; Groth, T. Self-assembled monolayers with different terminating groups as model substrates for cell adhesion studies. Biomaterials 2004, 25 (14), 27212730.

(15) Gimble, J. M.; Katz, A. J.; Bunnell, B. A. Adipose-derived stem cells for regenerative medicine. Circ. Res. 2007, 100 (9), 1249-1260.

(16) Liang, H.; Miranto, H.; Granqvist, N.; Sadowski, J. W.; Viitala, T.; Wang, B.; Yliperttula, M. Surface plasmon resonance instrument as a refractometer for liquids and ultrathin films. Sens. Actuators, B 2010, 149 (1), 212-220.

(17) Teixeira, R.; Reis, R. L.; Pashkuleva, I. Influence of the sulfation degree of glycosaminoglycans on their multilayer assembly with poly-1lysine. Colloids Surf., B 2016, 145, 567-575.

(18) Schwarzbauer, J. E.; DeSimone, D. W. Fibronectins, Their Fibrillogenesis, and In Vivo Functions. Cold Spring Harbor Perspect. Biol. 2011, 3 (7), a005041.

(19) Bloom, L.; Ingham, K. C.; Hynes, R. O. Fibronectin regulates assembly of actin filaments and focal contacts in cultured cells via the heparin-binding site in repeat III13. Mol. Biol. Cell 1999, 10 (5), $1521-1536$.

(20) Lehnert, M.; Gorbahn, M.; Rosin, C.; Klein, M.; Koper, I.; AlNawas, B.; Knoll, W.; Veith, M. Adsorption and Conformation Behavior of Biotinylated Fibronectin on Streptavidin-Modified TiOX Surfaces Studied by SPR and AFM. Langmuir 2011, 27 (12), 77437751.

(21) Mitsi, M.; Hong, Z. N.; Costello, C. E.; Nugent, M. A. Heparinmediated conformational changes in fibronectin expose vascular endothelial growth factor binding sites. Biochemistry 2006, 45 (34), 10319-10328.

(22) Hubbard, B.; Buczek-Thomas, J. A.; Nugent, M. A.; Smith, M. L. Heparin-dependent regulation of fibronectin matrix conformation. Matrix Biol. 2014, 34, 124-131.

(23) Yamada, K. M.; Kennedy, D. W.; Kimata, K.; Pratt, R. M. Characterization of Fibronectin Interactions with Glycosaminoglycans 
and Identification of Active Proteolytic Fragments. J. Biol. Chem. 1980, 255 (13), 6055-6063.

(24) Evington, J. R. N.; Luscombe, M.; Holbrook, J. J. Interaction of Plasma Fibronectin with Heparin and Heparan-Sulfate. Biochem. Soc. Trans. 1985, 13 (1), 243-244.

(25) Benecky, M. J.; Kolvenbach, C. G.; Amrani, D. L.; Mosesson, M. W. Evidence That Binding to the Carboxyl-Terminal Heparin-Binding Domain (Hep-Ii) Dominates the Interaction between Plasma Fibronectin and Heparin. Biochemistry 1988, 27 (19), 7565-7571.

(26) Altankov, G.; Grinnell, F.; Groth, T. Studies on the biocompatibility of materials: Fibroblast reorganization of substratum-bound fibronectin on surfaces varying in wettability. J. Biomed. Mater. Res. 1996, 30 (3), 385-391.

(27) Gugutkov, D.; Altankov, G.; Hernandez, J. C. R.; Pradas, M. M.; Sanchez, M. S. Fibronectin activity on substrates with controlled -OH density. J. Biomed. Mater. Res., Part A 2010, 92A (1), 322-331.

(28) Roach, P.; Farrar, D.; Perry, C. C. Interpretation of protein adsorption: Surface-induced conformational changes. J. Am. Chem. Soc. 2005, 127 (22), 8168-8173.

(29) Baugh, L.; Vogel, V. Structural changes of fibronectin adsorbed to model surfaces probed by fluorescence resonance energy transfer. $J$. Biomed. Mater. Res. 2004, 69A (3), 525-534.

(30) Rico, P.; Mnatsakanyan, H.; Dalby, M. J.; Salmerón-Sánchez, M. Material-Driven Fibronectin Assembly Promotes Maintenance of Mesenchymal Stem Cell Phenotypes. Adv. Funct. Mater. 2016, 26, 6563.

(31) Lin, M.; Wang, H.; Ruan, C.; Xing, J.; Wang, J.; Li, Y.; Wang, Y.; Luo, Y. Adsorption force of fibronectin on various surface chemistries and its vital role in osteoblast adhesion. Biomacromolecules 2015, 16 (3), 973-84.

(32) Llopis-Hernandez, V.; Cantini, M.; Gonzalez-Garcia, C.; Cheng, Z. A.; Yang, J.; Tsimbouri, P. M.; Garcia, A. J.; Dalby, M. J.; SalmeronSanchez, M. Material-driven fibronectin assembly for high-efficiency presentation of growth factors. Sci. Adv. 2016, 2 (8), e1600188.

(33) Baneyx, G.; Vogel, V. Self-assembly of fibronectin into fibrillar networks underneath dipalmitoyl phosphatidylcholine monolayers: Role of lipid matrix and tensile forces. Proc. Natl. Acad. Sci. U. S. A. 1999, 96 (22), 12518-12523.

(34) Faucheux, N.; Tzoneva, R.; Nagel, M.-D.; Groth, T. The dependence of fibrillar adhesions in human fibroblasts on substratum chemistry. Biomaterials 2006, 27, 234-245.

(35) Grinnell, F. Focal Adhesion Sites and the Removal of Substratum-Bound Fibronectin. J. Cell Biol. 1986, 103 (6), 26972706.

(36) Garcia, A. J.; Vega, M. D.; Boettiger, D. Modulation of Cell Proliferation and Differentiation through Substrate-dependent Changes in Fibronectin Conformation. Mol. Biol. Cell 1999, 10, 785-798.

(37) Tymchenko, N.; Nileback, E.; Voinova, M. V.; Gold, J.; Kasemo, B.; Svedhem, S., Reversible Changes in Cell Morphology due to Cytoskeletal Rearrangements Measured in Real-Time by QCM-D. Biointerphases 2012, 7 (1-4), DOI: 10.1007/s13758-012-0043-9.

(38) Fredriksson, C.; Kihlman, S.; Rodahl, M.; Kasemo, B. The piezoelectric quartz crystal mass and dissipation sensor: A means of studying cell adhesion. Langmuir 1998, 14 (2), 248-251.

(39) Fredriksson, C.; Khilman, S.; Kasemo, B.; Steel, D. M. In vitro real-time characterization of cell attachment and spreading. J. Mater. Sci.: Mater. Med. 1998, 9 (12), 785-788.

(40) Lord, M. S.; Modin, C.; Foss, M.; Duch, M.; Simmons, A.; Pedersen, F. S.; Besenbacher, F.; Milthorpe, B. K. Extracellular matrix remodelling during cell adhesion monitored by the quartz crystal microbalance. Biomaterials 2008, 29 (17), 2581-2587.

(41) Nimeri, G.; Fredriksson, C.; Elwing, H.; Liu, L.; Rodahl, M.; Kasemo, B. Neutrophil interaction with protein-coated surfaces studied by an extended quartz crystal microbalance technique. Colloids Surf., B 1998, 11 (5), 255-264.

(42) Nileback, E.; Enochson, L.; Altgarde, N.; Schnabelrauch, M.; Lindahl, A.; Svedhem, S.; Kunze, A. Acoustic monitoring of changes in well-defined hyaluronan layers exposed to chondrocytes. Analyst 2014, 139 (21), 5350-5353. 